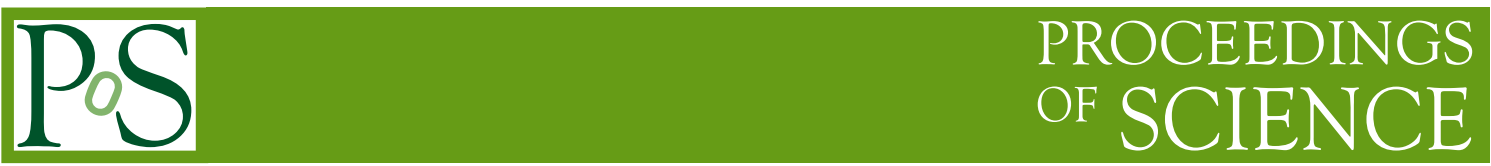

\title{
Performance of the CMS muon trigger system in proton-proton collisions at $13 \mathrm{TeV}$
}

\author{
Carlos Vico Villalba on behalf of the CMS Collaboration \\ Universidad de Oviedo, Oviedo, Spain \\ E-mail: vicocarlos@uniovi.es
}

During Run 2 (covering 2015-2018) the LHC achieved a maximum instantaneous luminosity of $2 \times 10^{34} \mathrm{~cm}^{-2} \mathrm{~s}^{-1}$ while delivering proton-proton collisions at $\sqrt{s}=13 \mathrm{TeV}$. The challenge for the trigger system of the CMS experiment is to reduce the event rate from about $40 \mathrm{MHz}$ to about $1 \mathrm{kHz}$. In this document, muon reconstruction and identification algorithms used during Run 2 and their improvements are presented. The new algorithms maintain the acceptance of the muon triggers at the same or even lower rate throughout the data-taking period despite the increasing number of additional proton-proton interactions in each LHC bunch crossing. We will focus on the single and double muon triggers with the lowest sustainable transverse momentum thresholds used by CMS. The efficiency is measured in a transverse momentum range from 8 to several hundred $\mathrm{GeV}$.

\footnotetext{
*** The European Physical Society Conference on High Energy Physics (EPS-HEP2021), *** *** 26-30 July $2021 * * *$

*** Online conference, jointly organized by Universität Hamburg and the research center DESY ***
} 


\section{Introduction}

Many important signatures studied at the CERN LHC involve muons, including final states from vector boson, B hadrons, Higgs bosons and even signatures used for searches of new physics beyond the standard model. Therefore, efficient reconstruction and identification of muons is crucial to the success of the CMS [1] physics program. During the Run 2 (years 2015-2018), the LHC delivered collisions at a center-of-mass energy of $13 \mathrm{TeV}$, and with a peak instantaneous luminosity of $2.1 \times 10^{34} \mathrm{~cm}^{-2} \mathrm{~s}^{-1}$. To cope with these harsh luminosity conditions, the CMS trigger system had to be in constant development, so that acceptable event rates for storage and analysis were maintained. This also lead to the necessity of improving the performance of the algorithms used in the reconstruction of physical objects. In this document we summarize the improvements and performance of the muon trigger reconstruction algorithms during Run 2.

\section{Improvements at the High Level Trigger}

The CMS trigger system [2] is divided in two levels: the Level-1 trigger (L1T), a hardwarebased system acting at collision time; and the high-level-trigger (HLT), which is software-based. The HLT muon reconstruction is performed in two steps: Level-2 (L2) and Level-3 (L3). At L2 muons are reconstructed using only information from the muon system. Then, this reconstruction is combined with information from the tracker system, which is incorporated at L3, in order to reconstruct the full trajectory of the muon. Lastly, a muon isolation requirement is applied, meant to separate signal-like muons, which are those produced after vector or Higgs boson decays, from those produced inside decays in flight and meson decays.

The L2 reconstruction, due to the muon chambers not being significantly affected by the increasing pileup, has been stable and has showed excellent performance during the whole Run 2. The $\mathrm{L} 3$ reconstruction, on the other hand, relies on good tracking detection, which is strongly affected by increasing pileup. Therefore, several improvements were implemented at this level in order to maintain optimal muon reconstruction performance. In 2016, the muon reconstruction was based on the usage of two different algorithms: the "cascade" and "tracker" algorithms. The combination of these two approaches was enough to reconstruct L3 muon objects with an efficiency of about 99.9\%. However, the tracker algorithm performance is strongly affected by harsher pileup, and therefore the main improvement at the L3 was the introduction of the "iterative" algorithm, which was designed to take advantage of an additional pixel layer added to the pixel detector in 2017 [3]. In addition, this new approach exploited the best aspects of both of the previous reconstruction algorithms. A more detailed description of the characteristics of these three algorithms can be found in [4].

\section{Results}

In Fig. 1a, the efficiency of L2 muon reconstruction is shown as a function of the muon $\mathrm{p}_{\mathrm{T}}$. For the three years of data taking, the average efficiency is found to be greater than $99.5 \%$ in the measured range. This measurement agrees with the expected independence of muon reconstruction performance at L2 in constantly increasing pileup environments. For the L3 reconstruction, a direct 
comparison of the performance of the iterative algorithm, and the logical OR of the cascade and tracker algorithms is shown in Fig. 1b, for different years of data taking. Both algorithms achieve reconstruction efficiencies above $99.5 \%$, being the cascade+tracker algorithm, used in 2016, more efficient than the approach followed in 2018, which used muons reconstructed with the iterative algorithm passing identification requirements.

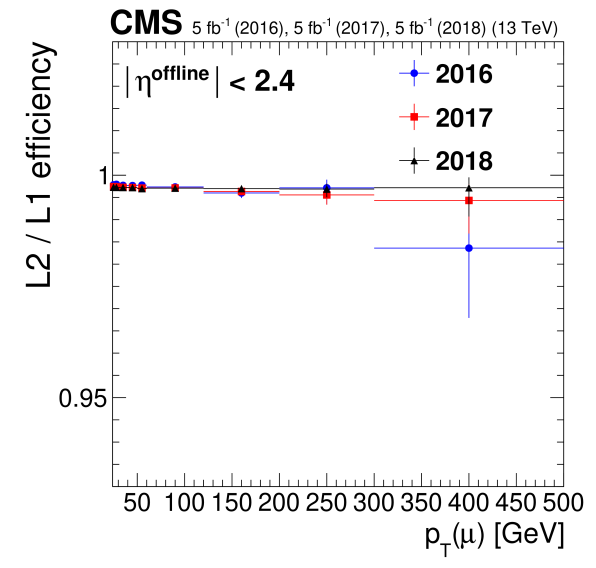

(a)

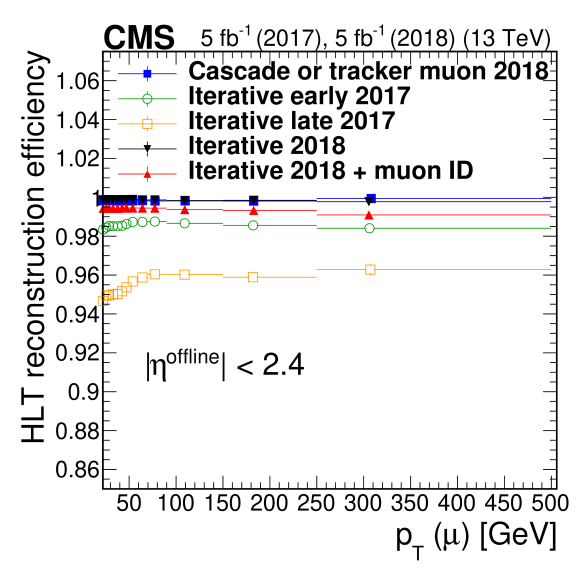

(b)

Figure 1: Efficiency for the L2 with respect to L1-muons, for the three years of data-taking and as a function of the muon transverse momentum (left). Efficiency for the iterative and the logical OR of the cascade and tracker muon algorithms, for 2018 data and two separate measurements from 2017, as a function of the $\mathrm{p}_{\mathrm{T}}$ (right) [4].

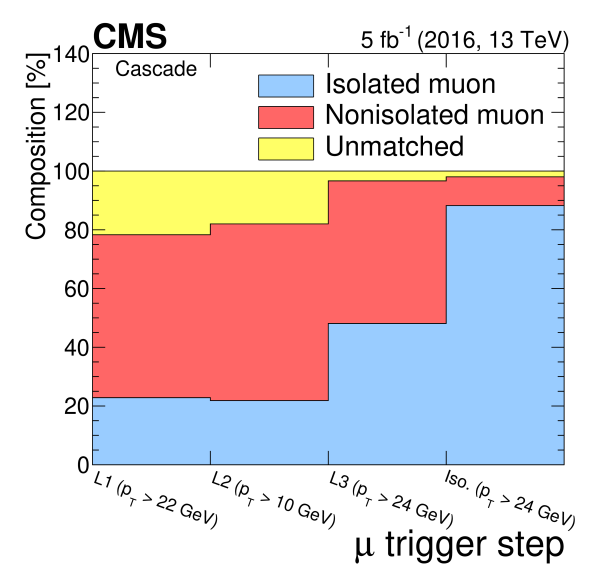

(a)

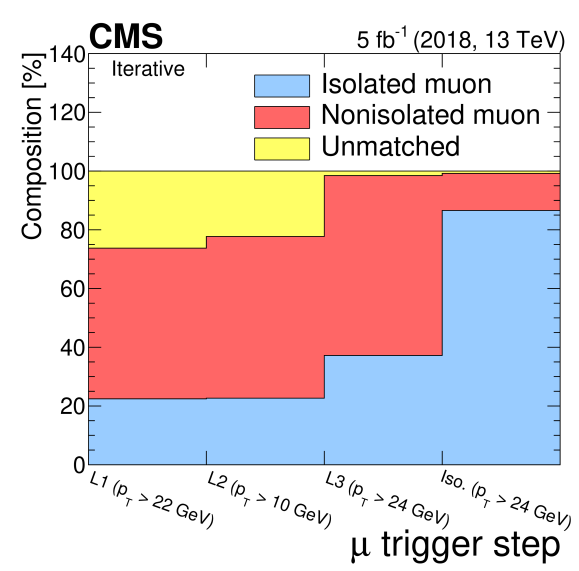

(b)

Figure 2: Composition of the selected muon sample for the 2016 (left) and 2018 (right) data. The enhancements on the L3 algorithms can be spotted on the considerable reduction in the amount of unmatched muons in the third and fourth bins, which decreases by a factor of about 3 [4].

The composition of L1, L2, L3, and isolated muons is shown in Figs. 2a and 2b, for 2016 and 2018 data, respectively. Comparing 2016 and 2018, one can see significant decrease in the fraction of unmatched muons at L3 by a factor of $\sim 3$, due to the improved reconstruction algorithm 
and the application of muon identification criteria. Finally, in order to evaluate and compare the performance between the previous and current HLT reconstruction approach, a single muon trigger with $\mathrm{p}_{\mathrm{T}}$ threshold of $24 \mathrm{GeV}$ and isolation requirements (IsoMu24) has been used to compute the processing time required for completing the full trigger sequence (reconstruction, identification and isolation). The cumulative average processing time is shown in Fig. 3. No difference between the years is observed in the reconstruction time for L2 muons. The $2016 \mathrm{~L} 3$ reconstruction algorithms (cascade and tracker muon) require around $29 \mathrm{~ms} /$ muon, while the average processing time in 2017 and 2018 (iterative) is 43 and $128 \mathrm{~ms} /$ muon, respectively.

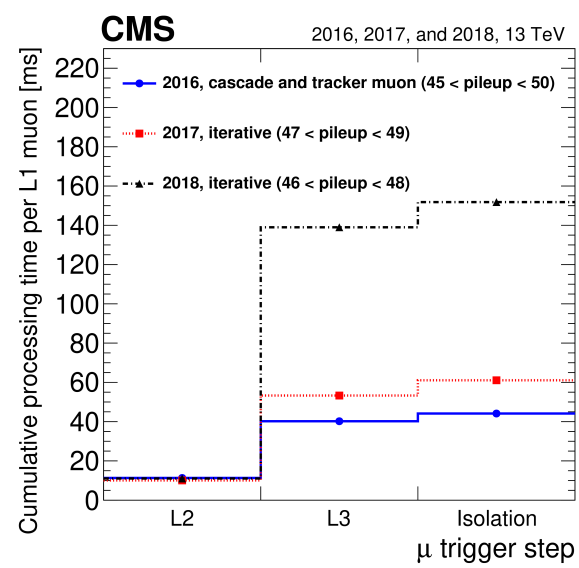

Figure 3: Cumulative average processing time for each of the trigger reconstruction steps for the IsoMu24 trigger. [4].

\section{Conclusions}

The continuous improvement in the muon reconstruction algorithms during Run2, as well as the upgrades of the L1-trigger, has contributed to maintain the overall efficiency for muon reconstruction at trigger level. Furthermore, the decreasing rate allows for using the same $p_{T}$ thresholds that were established for Run2, even though the luminosity conditions are expected to be much harsher. Because these improvements come at a cost of increased processing time needed to run the algorithms, this leaves room for further optimization for the upcoming LHC Run 3 with the goal to maintain the excellent physics performance while improving algorithmic efficiency.

\section{References}

[1] S. Chatrchyan et al. [CMS], JINST 3, S08004 (2008) doi:10.1088/1748-0221/3/08/S08004

[2] V. Khachatryan et al. [CMS], JINST 12 (2017) no.01, P01020 doi:10.1088/17480221/12/01/P01020 [arXiv:1609.02366 [physics.ins-det]].

[3] D. A. Matzner Dominguez et al. [CMS], doi:10.2172/1151650

[4] A. M. Sirunyan et al. [CMS], JINST 16 (2021), P07001 doi:10.1088/1748-0221/16/07/P07001 [arXiv:2102.04790 [hep-ex]]. 